This item was submitted to Loughborough's Research Repository by the author.

Items in Figshare are protected by copyright, with all rights reserved, unless otherwise indicated.

\title{
Mapping the structural order of laser-induced periodic surface structures in thin polymer films by microfocus beam grazing incidence small-angle $x$-ray scattering
}

\section{PLEASE CITE THE PUBLISHED VERSION}

https://doi.org/10.1021/am5074968

\section{PUBLISHER}

(c) American Chemical Society

\section{VERSION}

AM (Accepted Manuscript)

\section{PUBLISHER STATEMENT}

This work is made available according to the conditions of the Creative Commons Attribution-NonCommercialNoDerivatives 4.0 International (CC BY-NC-ND 4.0) licence. Full details of this licence are available at: https://creativecommons.org/licenses/by-nc-nd/4.0/

\section{LICENCE}

CC BY-NC-ND 4.0

\section{REPOSITORY RECORD}

Martin-Fabiani, Ignacio, Esther Rebollar, Mari-Cruz Garcia-Gutierrez, Daniel R. Rueda, Marta Castillejo, and Tiberio A. Ezquerra. 2017. "Mapping the Structural Order of Laser-induced Periodic Surface Structures in Thin Polymer Films by Microfocus Beam Grazing Incidence Small-angle X-ray Scattering". figshare. https://hdl.handle.net/2134/26694. 


\section{Mapping the Structural Order of Laser Induced}

\section{Periodic Surface Structures in Thin Polymer Films}

\section{by Microfocus Beam Grazing Incidence Small}

\section{Angle X-Ray Scattering}

Ignacio Martín-Fabiani, ${ }^{, 1}$ Esther Rebollar, ${ }^{2}$ Mari Cruz García-Gutiérrez, ${ }^{3}$ Daniel R. Rueda, ${ }^{3}$ Marta Castillejo, ${ }^{2}$ and Tiberio A. Ezquerra ${ }^{3}$

\footnotetext{
${ }^{1}$ Department of Physics, University of Surrey, Guildford GU2 7XH, United Kingdom

${ }^{2}$ Instituto de Química Física Rocasolano (IQFR-CSIC), Serrano 119, 28006 Madrid, Spain

${ }^{3}$ Instituto de Estructura de la Materia (IEM-CSIC), Serrano 121, 28006 Madrid, Spain
}

KEYWORDS. LIPSS, polymer, thin films, microfocus, GISAXS.

ABSTRACT. In this work we present an accurate mapping of the structural order of Laser Induced Periodic Surface Structures (LIPSS) in spin-coated thin polymer films, via a microfocus beam Grazing Incidence Small Angle X-Ray Scattering ( $\mu$ GISAXS) scan, GISAXS modelling and Atomic Force Microscopy imaging all along the scanned area. This combined study has allowed evaluating the effects on LIPSS formation due to nonhomogeneous spatial distribution of the laser pulse energy, mapping with micrometric resolution the evolution of the period and degree of structural order of LIPSS across the laser beam diameter in a direction perpendicular to the polarization vector. The experiments 
presented go one step further towards controlling nanostructure formation in LIPSS through a deep understanding of the parameters that influence this process.

\section{INTRODUCTION}

Controlling nanostructure formation in thin polymer films is a key aspect for the development of several applications in nanotechnology, including photovoltaic devices, ${ }^{1}$ biosensors ${ }^{2}$ and phase change memories. ${ }^{3}$ Among other interesting structuration techniques such as solutionbased methods ${ }^{4,5}$ or nanoimprint lithography, ${ }^{6,7}$ laser irradiation plays a key role in the development of nanostructures for a broad variety of purposes. ${ }^{8}$ Achieving control over the formation process of these nanostructures requires an appropriate selection of characterization techniques as well as an accurate and exhaustive study of the influence of laser irradiation parameters on the nanostructure formation processes. One of the leading techniques for the assessment of nanostructures is Grazing Incidence Small Angle X-Ray Scattering (GISAXS), whose reflection geometry is extremely sensitive to the surface characteristics of the sample. ${ }^{9}$ This, together with the possibility of probing different depths in the sample by changing the angle of incidence, makes GISAXS a powerful tool that has been used for the characterization of many surface and interface morphologies. ${ }^{10-12}$ In recent times, the introduction of microbeams for GISAXS characterization has improved the spatial resolution of the technique. ${ }^{13}$ Moreover, modelling of the experimental GISAXS diagrams has been accomplished using different software packages, ${ }^{14,15}$ providing valuable information to interpret the experimental results. ${ }^{16,17}$

Laser induced periodic surface structures (LIPSS) are generated by irradiation of solid surfaces by linearly polarized laser pulses of nanosecond (ns) to femtosecond (fs) duration. In the ns regime the LIPSS period $(L)$ is close to the laser irradiation wavelength, ${ }^{18}$ and is described by the expression $L=\lambda /(n-\sin \theta) .{ }^{19}$ Here $\lambda$ is the laser wavelength illuminating the 
surface of a material of refraction index $n$ at an incidence angle $\theta$ with respect to the normal to the sample plane. LIPSS formation is explained on the basis of the interference between the incoming and the surface-scattered waves together with a feedback mechanism. ${ }^{20,21}$ In the case of LIPSS in thin polymer films, using irradiation fluences well below the ablation threshold of the material, recent studies combining experimental GISAXS and modelling with Atomic Force Microscopy (AFM) have proved that it is possible to correlate the degree of structural order with the laser irradiation parameters, namely number of pulses and fluence, using both $\mathrm{ns}^{22}$ and fs laser pulses. ${ }^{23}$ In these studies, where the generated LIPSS were parallel to the laser polarization vector, the laser beam was delimitated by an iris, and it was assumed that the fluence was constant in the whole irradiated area. However, the nonhomogeneous spatial distribution of the laser pulse energy over the irradiated spot may play an important role on the generation of LIPSS and this effect has not been studied up to now. Such a study can provide an in depth knowledge of the formation of these structures, allowing optimization for singular applications as functional substrates. ${ }^{24}$

In this work we present an accurate mapping of the structural order of LIPSS in thin polymer films, via GISAXS with a microfocus beam, along a diameter of the laser irradiated area in steps of $25 \mu \mathrm{m}$. AFM imaging and GISAXS modelling have been performed as well, all along the scanned diameter in a direction perpendicular to the laser polarization vector and thus orthogonal to the LIPSS alignment axis. The results and conclusions derived are of high importance in order to control nanostructure formation and in all the applications where LIPSS are involved.

\section{EXPERIMENTAL SECTION}

The model polymer chosen for this work was poly (bisphenol A carbonate) (PBAC), Lexan ML3021A, SABIC I-P (Innovative Plastics). Thin polymer films were prepared by spin 
coating on silicon wafers (100) (Wafer World Inc.). The wafers were previously cleaned with acetone and isopropanol. PBAC was dissolved in chloroform (Riedel-de Haën, 99\%) with a concentration of $30 \mathrm{~g} / \mathrm{L}$. A fixed amount of $0.1 \mathrm{~mL}$ of polymer solution was instantly dropped by a syringe on a square (typically $2 \times 2 \mathrm{~cm}^{2}$ ) silicon substrate placed in the center of a rotating metallic horizontal plate. A rotation speed of $2380 \mathrm{rpm}$ was kept during $30 \mathrm{~s}$, obtaining spin-coated polymer films with a thickness of about $150 \mathrm{~nm}$. Laser irradiation was carried out in ambient air under normal incidence, with the linearly polarized output of a Qswitched Nd:YAG laser (Quantel Brilliant B, pulse duration $\tau=5$ ns full width halfmaximum) at a repetition rate of $10 \mathrm{~Hz}$. The fourth harmonic at $266 \mathrm{~nm}$ was used for the experiments, since at this wavelength PBAC absorbs efficiently. ${ }^{25,26}$ The average fluences of irradiation were determined by measuring the laser energy in front of the sample with a joulemeter (Gentec-E, QE25SP-H-MB-D0), and considering $9 \mathrm{~mm}$ as the diameter of the laser spot. The spin-coated polymer films were irradiated with 1200 pulses at an average fluence of $14 \mathrm{~mJ} / \mathrm{cm}^{2}$. The irradiated area was characterized using atomic force microscopy (AFM, Nanoscope V, Bruker) in tapping mode. AFM images were analyzed using the software Nanoscope Analysis 1.40. Irradiated areas were also characterized by microfocus beam grazing incidence X-ray scattering at small angle, $\mu$ GISAXS, using the facilities of the P03 beamline at PETRA III [Deutsches Elektronen Synchrotron (DESY), Hamburg, Germany]. ${ }^{27}$ The experimental setup for $\mu$ GISAXS is presented in Fig. 1. The sample is positioned horizontal and the incoming and reflected beams define the vertical scattering plane. Both scattering and sample planes intersect the detector along the meridian and the horizon lines, respectively, which are the reference to measure the out of scattering plane $(\omega)$ and exit $\left(\alpha_{f}\right)$ angles. The information can be interpreted on the basis of the two orthogonal scattering vectors $q_{z}=(2 \pi / \lambda)\left(\sin \alpha_{i}+\sin \alpha_{f}\right)$ and $q_{y}=(2 \pi / \lambda) \sin \omega \cos \alpha_{f}$, with $\lambda$ being the wavelength of the $\mathrm{X}$ ray beam and $\alpha_{i}$ and $\alpha_{f}$ the incidence and exit angles. These vectors 
provide information about structural correlations perpendicular and parallel to the film plane, respectively. $\mu$ GISAXS images were analyzed by using the software Fit2D. ${ }^{28}$ An X-ray wavelength of $\lambda=0.09764 \mathrm{~nm}$, with a beam size (h x v) of $20 \times 13 \mu \mathrm{m}^{2}$, was used in our experiments. The scattered intensity was recorded by a Pilatus detector of $487 \times 619$ pixels with a resolution of $172 \mu \mathrm{m}$ per pixel, and a sample-to-detector distance of $4.175 \mathrm{~m}$. Samples were positioned to ensure that the beam was parallel to the LIPSS main axis. A step scan (25 $\mu \mathrm{m}$ resolution) was performed along a diameter of the irradiated area, in a direction perpendicular to the laser polarization vector and thus orthogonal to the LIPSS axis. Acquisition times of $3 \mathrm{~s}$ were used in order to minimize the X-ray radiation damage inflicted on the sample.

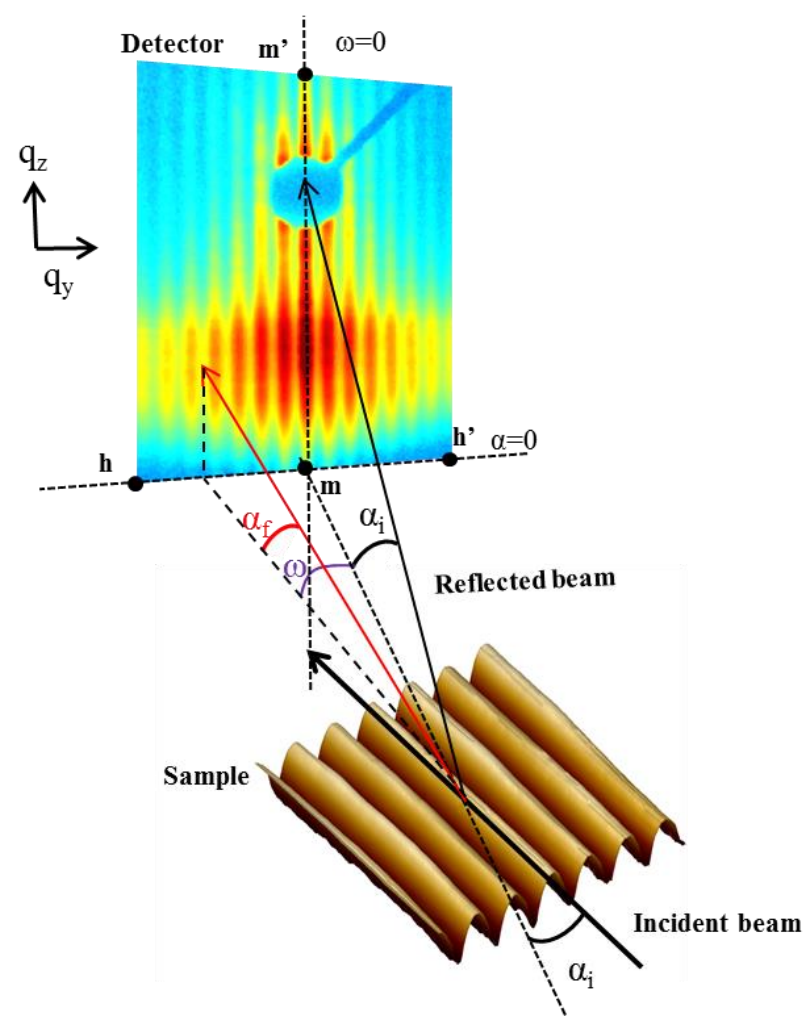

Figure 1. Schematic view of a GISAXS experiment. The scattering plane, containing both the direct and the specular beams, intersects the 2D detector along the meridian, $\mathrm{m} \_\mathrm{m}$ ' line, of the GISAXS pattern. The horizon, $h$ _h' line, is the intersection between the sample plane 
and the plane of the 2D detector, which are perpendicular to each other. Each point on the GISAXS pattern can be characterized by the exit angle, $\alpha_{f}$, and the out of scattering plane angle, $\omega . \alpha_{i}$ represents the angle of incidence of the X-ray beam on the sample.

It is important to mention that some imprecisions in the correlation between AFM, $\mu$ GISAXS and local energy measurement positions might be possible. The experimental error is not only present in the local energy profile determination, but also in the position of the x-ray beam on the sample and in the AFM image position. In addition to this, the fact that the in the GISAXS experiments we are averaging over a strip rather than a line implies that we are probing regions with different local laser energy at the same time in the direction of the x-ray beam. However, the agreement is quite good as we will prove throughout the manuscript.

\section{EXPERIMENTAL RESULTS}

Fig. 2a shows an optical image of the irradiated polymer thin film after carrying out the $\mu$ GISAXS experiments. Two vertical blue stripes are visible at positions $-4.5 \mathrm{~mm}$ and $5 \mathrm{~mm}$, corresponding to beginning and end of the $\mu$ GISAXS scan performed. These marks were intentionally produced by letting the X-ray beam shutter open for $200 \mathrm{~s}$ after the experiments were over. It is also possible to see a weaker blue stripe around $2 \mathrm{~mm}$, which is due to radiation damage inflicted to the sample while aligning it for the $\mathrm{X}$-ray measurements. To characterize the energy profile of the laser beam, the energy was measured using a straight knife-edge which was translated through the beam in steps of $250 \mu \mathrm{m}$ using a translation stage, and subsequent first derivative calculation of the dependence obtained. The spatial energy distribution of the laser spot along the direction of the $\mu$ GISAXS scan is presented in Fig. 2b. As one can see, the local energy is not completely uniform and depends on the position on the sample. The local laser energy profile in the direction perpendicular to the GISAXS scan (parallel to the X-ray beam) reveals as well an inhomogeneous spatial energy 
distribution, as it is shown in Fig. 2c. These differences between local energies may result in inhomogeneity in the LIPSS formation, thus creating regions with different period, height and degree of structural order. ${ }^{22}$

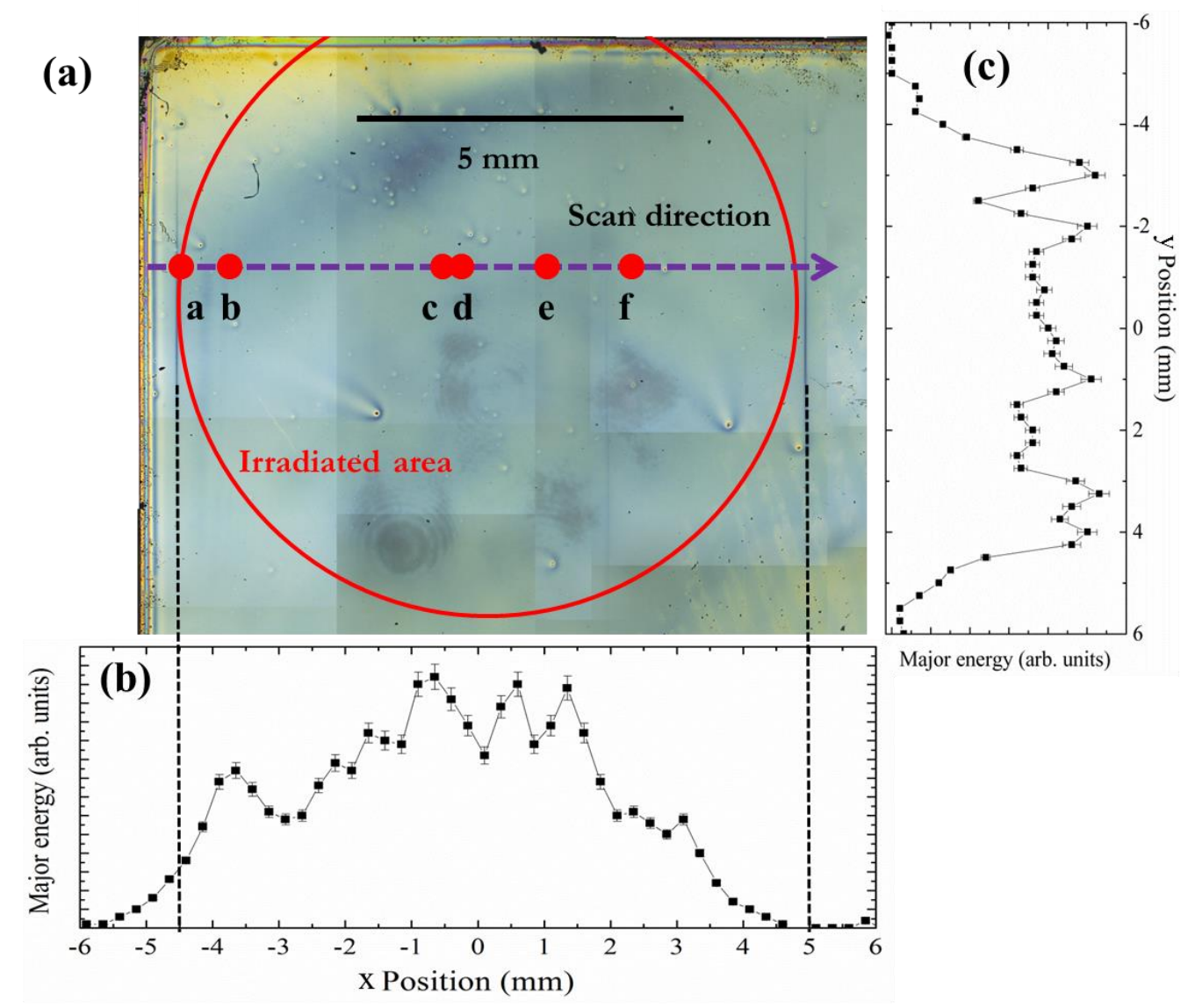

Figure 2. (a) Optical microscopy image of the laser-irradiated area (red circle) on the thin polymer film, showing the lateral marks (vertical dashed lines) that define the beginning and end of the scan performed along the direction of the horizontal dashed arrow in the $\mu$ GISAXS experiments. The X-ray beam is in the vertical direction. The red spots, labeled a-f, indicate selected scan positions where $\mu$ GISAXS diagrams shown in Fig. 3 were acquired. (b) Local energy profile of the laser beam used for irradiation along the direction of the $\mu$ GISAXS scan (horizontal dashed arrow), which is perpendicular to the laser polarization vector and to the axis of the generated LIPSS. (c) Local energy profile of the laser beam used for irradiation along the direction perpendicular to the GISAXS scan direction at $\mathrm{x}=0$. 
The $\mu$ GISAXS experiments reveal significant differences in the surface structuring of the thin polymer film depending on the position along the scan. Fig. 3 presents $\mu$ GISAXS diagrams acquired at selected regions of the sample. These regions are identified as labelled red spots in Fig. 2a. At the beginning of the scan corresponding to a low local energy range (Fig. 2b) the $\mu$ GISAXS diagrams (position a, Fig. 3a) do not show any sign of LIPSS formation. This fact can be also visualized by representing a cut at an exit angle of $\alpha_{f}=0.13^{\circ}$ (marked by a dashed black line in Fig. 3a), close to the critical angle of the polymer, as represented in Fig. 4a. When inspected by AFM, this area corresponds to a region where no LIPSS have been formed, as it is observed in Fig. 5a and further demonstrated by the depth profile shown below. Moving forward, to position $b$, there is a narrow area located at around $-3.9 \mathrm{~mm}$ where clear rods are detected out of the meridian (Fig. 3b), indicating the existence of a strong structural correlation in the direction parallel to the scan direction. In a first approach, the period $L$ of the ripples can be determined from the $\mu$ GISAXS cut (Fig. $4 \mathrm{~b}$ ) using the expression $L=2 \pi / q_{y}{ }^{\max }$, where $q_{y}{ }^{\max }$ is the reciprocal scattering vector corresponding to the first intensity maximum next to $\omega=0$. In this case the value obtained is significantly shorter than the laser wavelength used, $L_{b}$ GISAXS $=182 \pm 9 \mathrm{~nm}$. AFM images taken in this area (Fig. 5b) confirm that polymer ripples have been generated on the surface of the film, having a period in agreement with the value obtained from the $\mu$ GISAXS measurement (see Table 1). 


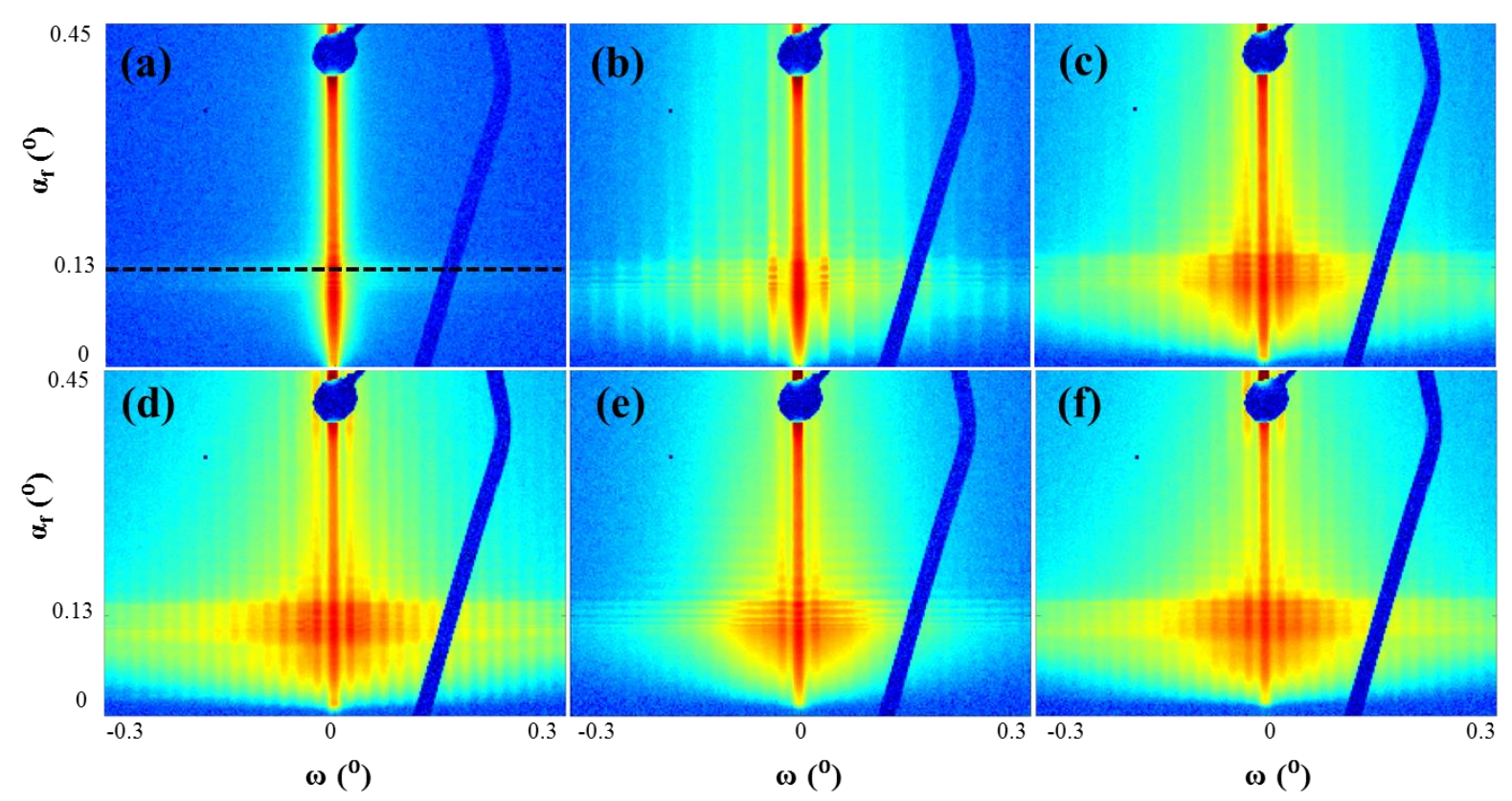

Figure 3. Selected $\mu$ GISAXS diagrams of PBAC irradiated at $266 \mathrm{~nm}$ with 1200 pulses at different positions on the irradiated polymer surface as shown in Fig. 2a: (a) $-4.5 \mathrm{~mm}$, (b) -3.9 $\mathrm{mm}$, (c) $-0.5 \mathrm{~mm},(\mathrm{~d})-0.4 \mathrm{~mm}$, (e) $0.8 \mathrm{~mm}$ and (f) $2.4 \mathrm{~mm}$. The dashed black line in (a) represents the exit angle of $\alpha_{f}=0.13^{\circ}$ where the cuts in Fig. 4 where obtained from. 

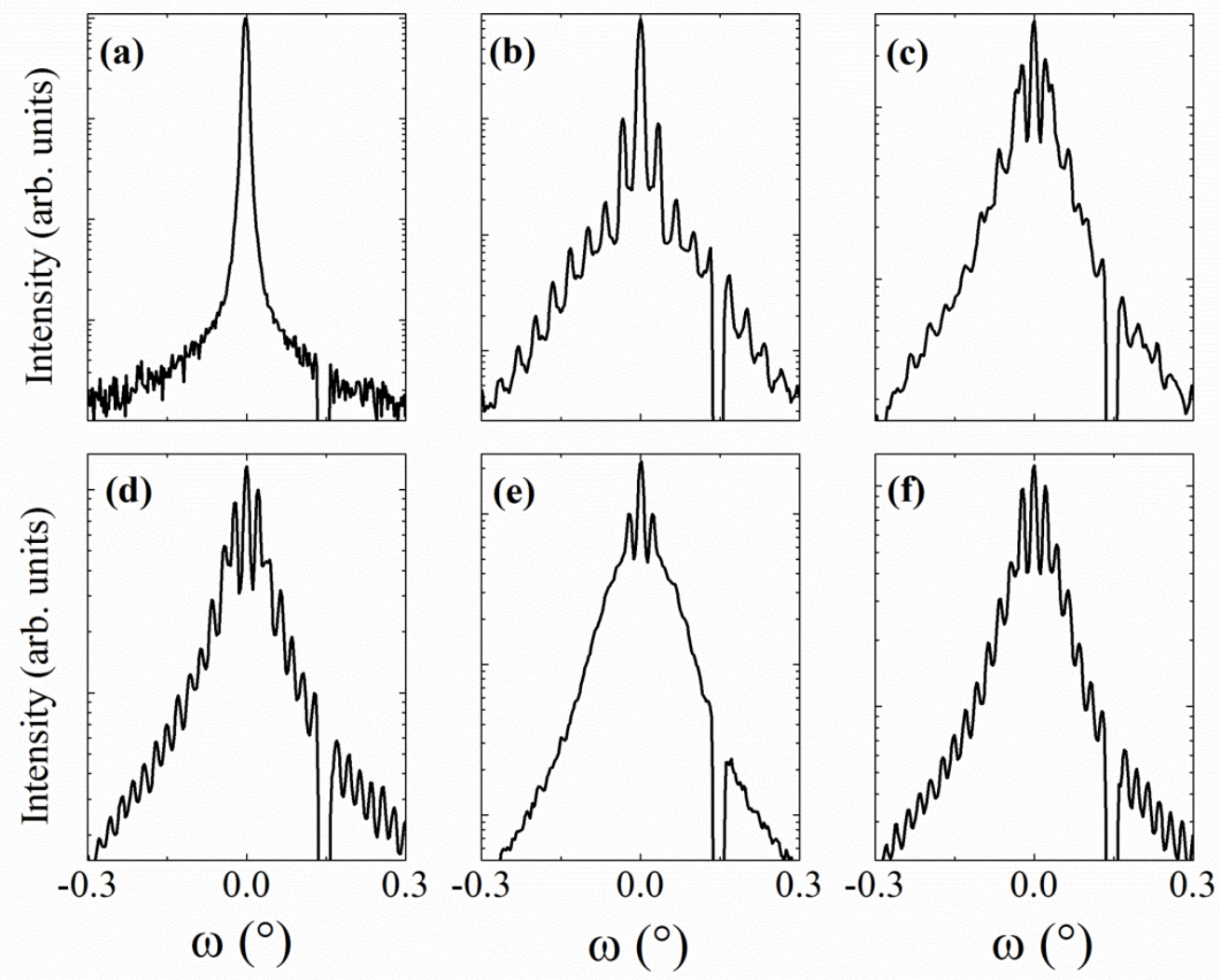

Figure 4. Intensity profiles at an exit angle $\alpha_{f}=0.13^{\circ}$ (black dashed line in Fig. 3a) corresponding to the GISAXS diagrams shown in Figure 3, according with the labelling of Fig. 2: (a) $-4.5 \mathrm{~mm},(\mathrm{~b})-3.9 \mathrm{~mm}$, (c) $-0.5 \mathrm{~mm}$, (d) $-0.4 \mathrm{~mm}$, (e) $0.8 \mathrm{~mm}$ and (f) $2.4 \mathrm{~mm}$. 

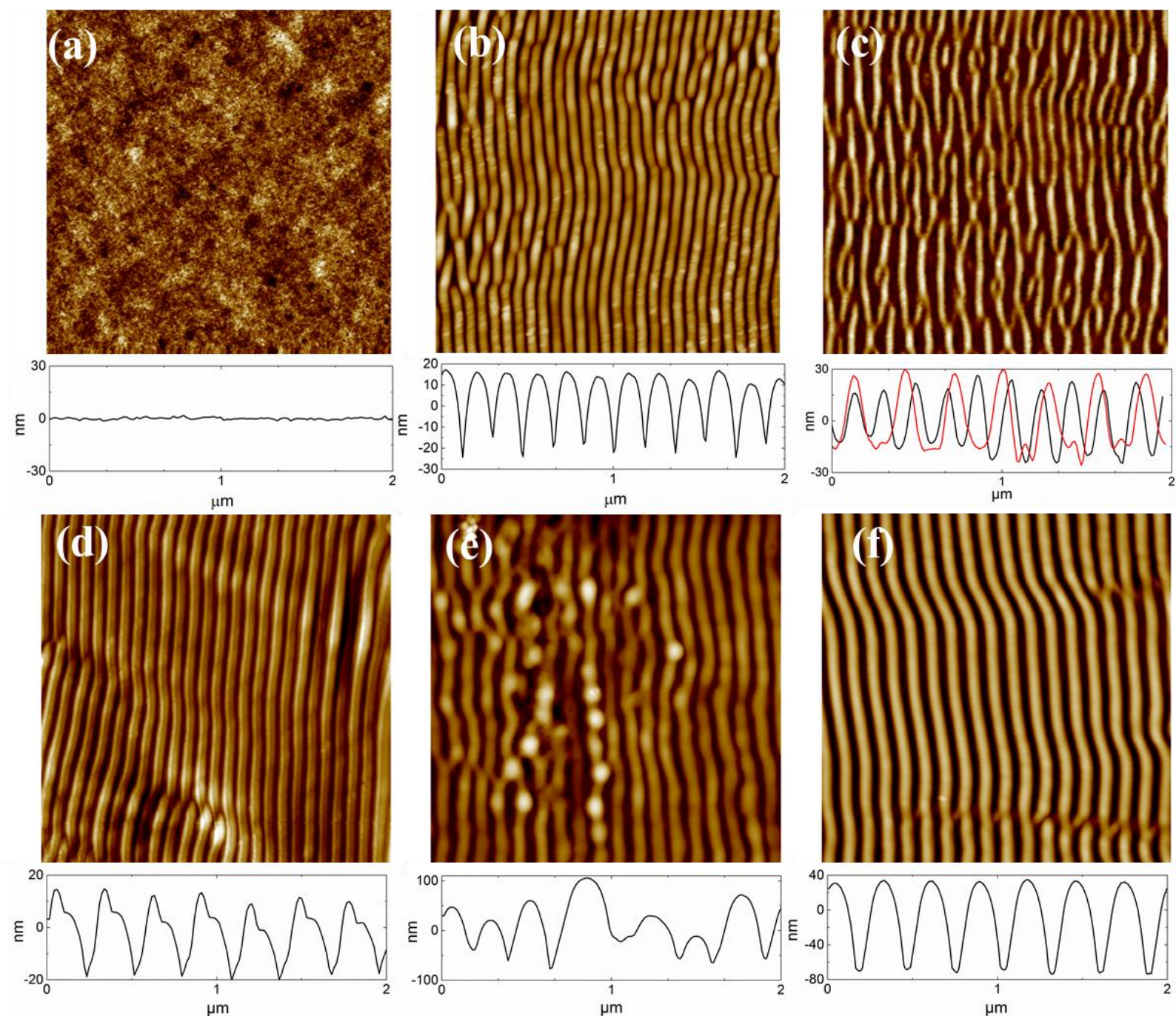

Figure 5. AFM topography images $\left(5 \times 5 \mu \mathrm{m}^{2}\right)$ of PBAC irradiated with 1200 pulses at selected regions of the laser irradiated spot according with the labelling of Fig. 2: (a) -4.5 mm, (b) $-3.9 \mathrm{~mm}$, (c) $-0.5 \mathrm{~mm}$, (d) $-0.4 \mathrm{~mm}$, (e) $0.8 \mathrm{~mm}$ and (f) $2.4 \mathrm{~mm}$. Height profiles along a $2 \mu \mathrm{m}$ line perpendicular to the ripples are shown below every image. Black and red lines below (c) correspond, respectively to cuts in regions with short and large period.

Table 1. Geometric parameters of the LIPSS generated on PBAC films and values used for the simulations of GISAXS patterns: width $(2 R)$, height $(H)$, and length $(W)$ of the box considered as scatterer, period $(L)$ and paracrystalline disorder parameter $(g)$. Gaussian distributions were assumed for $R$ and $H$ with $\sigma_{R} / R$ and $\sigma_{H} / H$ ca. 0.1 . 


\begin{tabular}{|c|c|c|c|c|c|c|c|}
\hline Label & $\begin{array}{c}\text { Position } \\
(\mathbf{m m})\end{array}$ & $\begin{array}{c}\boldsymbol{L}_{\text {GISAXS }} \\
(\mathbf{n m})\end{array}$ & $\begin{array}{c}\boldsymbol{L}_{\text {AFM }} \\
(\mathbf{n m})\end{array}$ & $\begin{array}{c}\mathbf{2 R} \\
(\mathbf{n m})\end{array}$ & $\begin{array}{c}\boldsymbol{H} \\
(\mathbf{n m})\end{array}$ & $\begin{array}{c}\boldsymbol{W} \\
(\mathbf{n m})\end{array}$ & $\boldsymbol{g}$ \\
\hline $\mathrm{a}$ & -4.5 & - & - & - & - & - & - \\
\hline $\mathrm{b}$ & -3.9 & $182 \pm 9$ & $191 \pm 16$ & 104 & 30 & 1000 & 0.0235 \\
\hline $\mathrm{c}$ & -0.5 & $\begin{array}{c}169 \pm 10 \\
263 \pm 8\end{array}$ & $\begin{array}{c}179 \pm 10 \\
278 \pm 9\end{array}$ & - & - & - & - \\
\hline $\mathrm{d}$ & -0.4 & $263 \pm 8$ & $276 \pm 11$ & 136 & 38 & 1000 & 0.0114 \\
\hline $\mathrm{e}$ & 0.8 & $263 \pm 8$ & $270 \pm 18$ & 180 & 45 & 900 & 0.0456 \\
\hline $\mathrm{f}$ & 2.4 & $263 \pm 8$ & $274 \pm 10$ & 122 & 67 & 1000 & 0.0114 \\
\hline
\end{tabular}

Moving further along the scan direction towards the center of the laser spot, after an unstructured region i.e. showing GISAXS without any defined rods, clear signs of structure formation are again revealed in the $\mu$ GISAXS diagrams at scan positions between $-1.5 \mathrm{~mm}$ and $0.5 \mathrm{~mm}$ (Fig. 3c). In this case the diagrams indicate the presence of structures with two different periods. This is confirmed when inspecting the corresponding angular cut in Fig. 4c, which evidences the presence of both structures with a period close to the laser wavelength $L_{c, \text { large }}^{\text {GISAXS }}=263 \pm 8 \mathrm{~nm}$ and a shorter one $L_{c, \text { short }}^{\text {GISAXS }}=169 \pm 10 \mathrm{~nm}$. AFM characterization supports as well the presence of two LIPSS populations (Fig. 5c). The topography profiles perpendicular to the structures in a region with short small period (black cut below Fig. 5c) and in another region with large period (red cut below Fig. 5c) evidence the difference between the two populations. The specific values of the two periods obtained by AFM are again in agreement with the ones obtained by GISAXS (Table 1).

Following the region where two different ripple periods coexist, the scan inspects the central region of the laser spot, between 0.5 and $3 \mathrm{~mm}$, (positions d, e, f in Fig. 2a), where the laser energy reaches maximum values (see Fig. 2b). Here the $\mu$ GISAXS patterns show well defined correlation peaks (Figs. 3d, 3e and 3f) and a single and constant period as determined 
by the cuts at $\alpha=0.13^{0}$ (Figs. 4d, 4e, and 4f) with value $L_{d, e, f}$ GISAXS $=263 \pm 8 \mathrm{~nm}$. However, the AFM images suggest that the degree of structural order changes spatially in this region. Figs. 5d, 5e and $5 \mathrm{f}$ and the corresponding topographic profiles show polymer ripples with a period in agreement with the one calculated from the $\mu$ GISAXS diagrams (see Table 1), but with different degrees of structural order. These observations will be discussed further in the following section. After reaching the local laser energy maximum and moving further along the irradiated area, the $\mu$ GISAXS patterns are similar to those shown in Fig. $3 c$ presenting again two different periods and indicating certain symmetry in the formation of structures with respect to the center of the laser beam.

\section{MODELLING AND DISCUSSION}

Mapping the formation of LIPSS in our experiments can be carried out by considering the structural information obtained by $\mu$ GISAXS and AFM and establishing a correlation with the laser energy profile (see Fig. $2 \mathrm{~b}$ and Fig. 6a). In order to achieve this goal, the spatial evolution of the LIPSS period along the $\mu$ GISAXS scan has been represented in Fig. $6 \mathrm{~b}$. In the low local energy region, at the beginning of the scan, no ripples are observed. This is in agreement with the known fact of the existence of a threshold in fluence needed to make LIPSS formation effective. ${ }^{25}$ After this initial region, LIPSS formation with a period smaller than the laser wavelength is detected in a narrow area around position $-3.9 \mathrm{~mm}$ (Fig. 6a). This region coincides with a secondary peak in the local laser energy (Fig. 2b). It has been previously reported that LIPSS period in polymers increases with increasing fluence until a certain value, above which the LIPSS period reaches the laser wavelength. Thus, it can be concluded that in this area the local laser energy is above the threshold for PBAC, but not high enough to generate structures with a period close to the laser irradiation wavelength. Following the scan, the local energy seems to go below the threshold and there are no signs 
of uniform LIPSS formation. Moving towards the center, another structured area is reached coinciding with an increase in the local laser pulse energy (Fig. 6a and b). In this case we find a region where two different LIPSS periods coexist, being the larger one close to the laser wavelength. We consider that this coexistence is an intermediate step in the formation of the final LIPSS, with period close to the laser wavelength. In Fig. 5c it is possible to see how the structures with small period converge in pairs into another type of ripple with larger period, closer to the laser wavelength. At this point it is important to remember that the local laser intensity is not only inhomogeneous in the GISAXS scan direction (Fig. 2b) but also in the perpendicular direction as it is shown in Fig. 2c. As a consequence, LIPSS with different periods can be formed along the x-ray beam direction for an $\mathrm{x}$ position fixed value. The $\mu$ GISAXS footprint, although is micrometric in the scan direction $(20 \mu \mathrm{m})$, due to the small incidence angle $\left(0.4^{\circ}\right)$ is in the order of $\mathrm{mm}$ in size in the direction perpendicular to the scan. Thus in the direction of the X-ray beam, a fairly long stripe is averaged in the $\mu$ GISAXS measurements, including regions with different periodicities which contribute to the scattering intensity. In the zone of highest local energy, close to the beam center (see Fig. 6a), LIPSS with a single period are formed. In this region the period reaches a plateau value that remains close to that of the laser wavelength (see Fig. 6b). However, AFM images taken in this area reveal structural changes. As an example, in Fig. 5e the ripples appear remarkably distorted and droplets of material start to form, as an indication of too high local laser energy. 


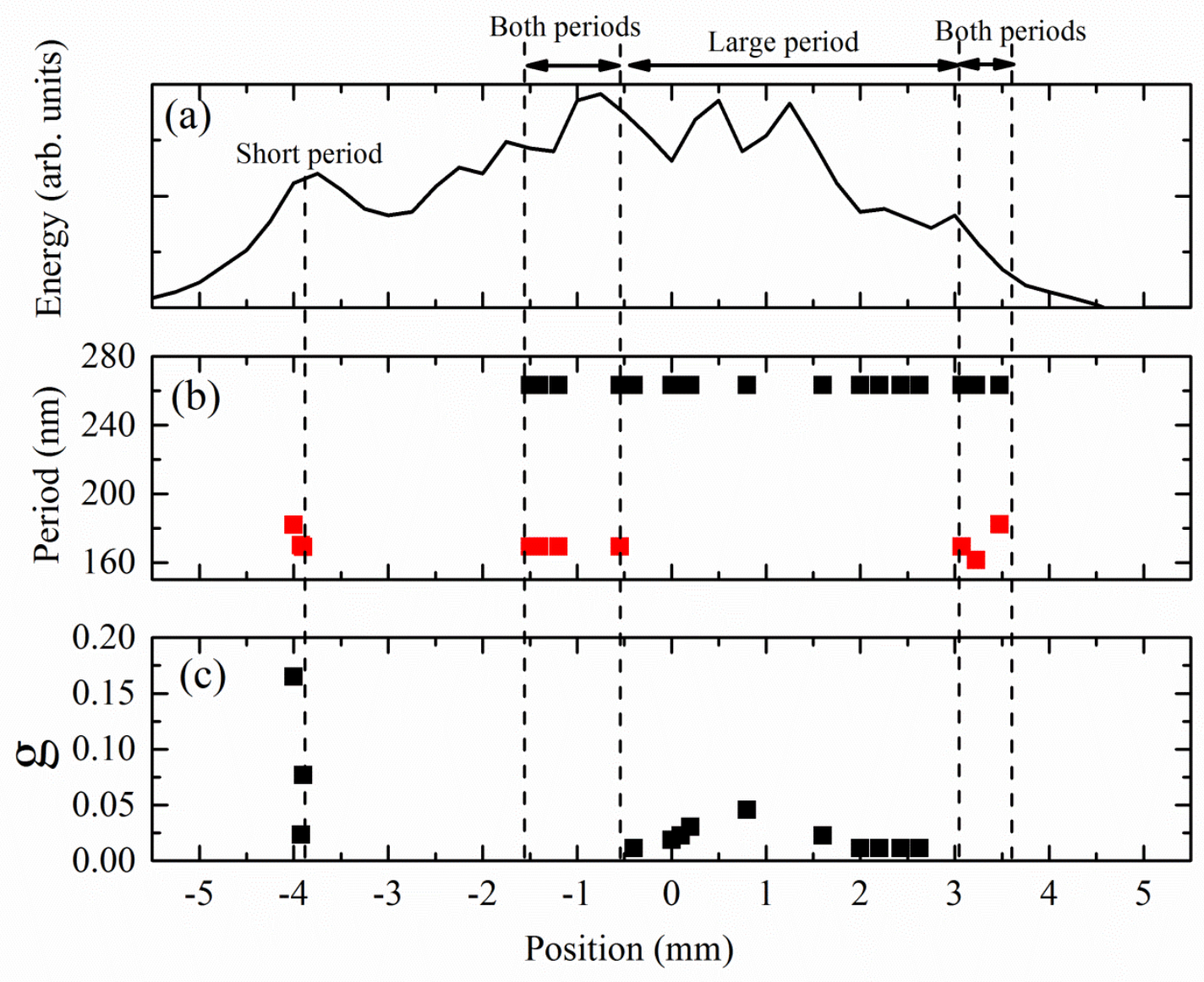

Figure 6. (a) Local energy profile of the laser beam along a diameter, perpendicular to the polarization vector and parallel to the scanning direction of the $\mu$ GISAXS experiments. This plot has been shown before in Fig. 2b. (b) LIPSS period as determined by GISAXS, with the red squares corresponding to the small period and the black squares corresponding to the large period close to the laser wavelength of $266 \mathrm{~nm}$. (c) Paracrystalline disorder parameter $g$ as determined by modelling of the GISAXS patterns.

In order to quantify the structural changes along the $\mu$ GISAXS scan we carried out modelling of the experimental diagrams by using the IsGISAXS software. ${ }^{14}$ The distorted wave Born approximation (DWBA) was applied, considering an array of polymer boxes standing on the polymer film. The values of the refraction indexes $(n=1-\delta+i \beta)$ for both box and polymer substrate were considered to be similar, $\delta=3.3 \times 10^{-6}$ and $\beta=5.1 \times 10^{-9}$. 
The dimensions of the boxes used to model the nanostructures are those determined by AFM, i.e., width $(2 R)$, height $(H)$, length $(W)$ and period $(L) .2 \mathrm{R}$ was determined by measuring the full width at half height of the ripples, obtained from height profiles perpendicular to their main axis. Up to 20 measurements are averaged for each geometric value given, and their typical deviation is used to calculate the statistical error.

$\mathrm{W}$ was estimated as an average measure of the length along which the ripples maintain a certain direction before changing it, as measured from AFM images. Box dimensions used to simulate the GISAXS patterns shown in Figure 7 are listed in Table 1. The next assumption was to consider that the boxes were in a one-dimensional paracrystalline lattice, where the long-range order disappears gradually in a probabilistic way. ${ }^{29}$ The probability of finding a particle at a distance $L$ is defined by a Gaussian function $p(\mathrm{x})$ :

$$
p(x)=\frac{1}{\sigma \sqrt{2 \pi}} \exp \left[-\frac{(x-L)^{2}}{2 \sigma^{2}}\right]
$$

As the paracrystalline disorder parameter $g=\sigma / L$ increases the lattice disorder increases, while for very small $g$ values, a $1 \mathrm{D}$ crystalline lattice is obtained. Fig. 7a and c show the experimental and modelled GISAXS diagrams for regions $b$, e and $f$ in the sample (defined in Fig. 2a). For the sake of comparison, the corresponding GISAXS intensity profiles at an exit angle of $\alpha_{f}=0.13^{\circ}$, normalized to the first correlation peak have been included in Fig. $7 \mathrm{~b}$. The modelling confirms that LIPSS can be well-described considering a one dimensional paracrystalline lattice and that the local laser energy has an influence on their structural order. It is worth remarking that the simulations show crystal truncation rods (CDR) whereas in the experimental data the intensity in the $q_{z}$ direction is modulated. This fact could be explained assuming that the LIPSS height distribution in the small region probed by the beam is very narrow, and will be investigated in future studies. By plotting the paracrystalline disorder 
parameter $g$ in terms of the scan position it is possible to map the structural order of the sample, as it is shown in Fig. 6c. Even in the very narrow region, where only short period LIPSS are present (around $-3.9 \mathrm{~mm}$ ), it is possible to detect variations of structural order depending on the local laser energy. Here the $g$ values are low, indicating that the degree of structural order of the short period LIPSS is high. Modelling was restricted to the GISAXS patterns with single periodicity. In the centre of the laser irradiated spot, where LIPSS period is fairly constant, it was possible to accurately determine the spatial dependence of the LIPSS structural order. As it can be seen in Fig. 6c, the differences in structural order observed by AFM in the high energy region are indeed related to regions with changing paracrystalline disorder parameter. At the beginning of this region $(-0.5 \mathrm{~mm}) \mathrm{g}$ is very low, denoting high structural order, and then increases smoothly until it reaches a maximum around $1 \mathrm{~mm}$. Subsequently, $g$ decreases again to stabilize at the same value calculated at the beginning of the high energy region. We associate this middle region, where the LIPSS display lower degree of structural order, to a region where the local laser energy is too high, as we already mentioned in the experimental results section when discussing the corresponding AFM images. This implies that combining $\mu$ GISAXS scan and modelling, together with the AFM characterization, we are able to map the structural order of LIPSS at a very fine level. 


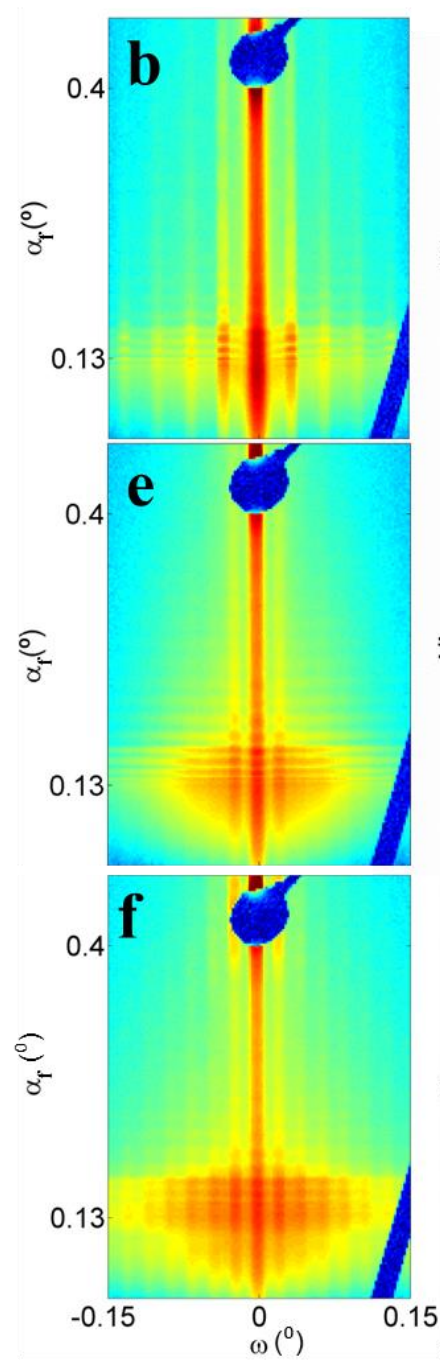

(a)
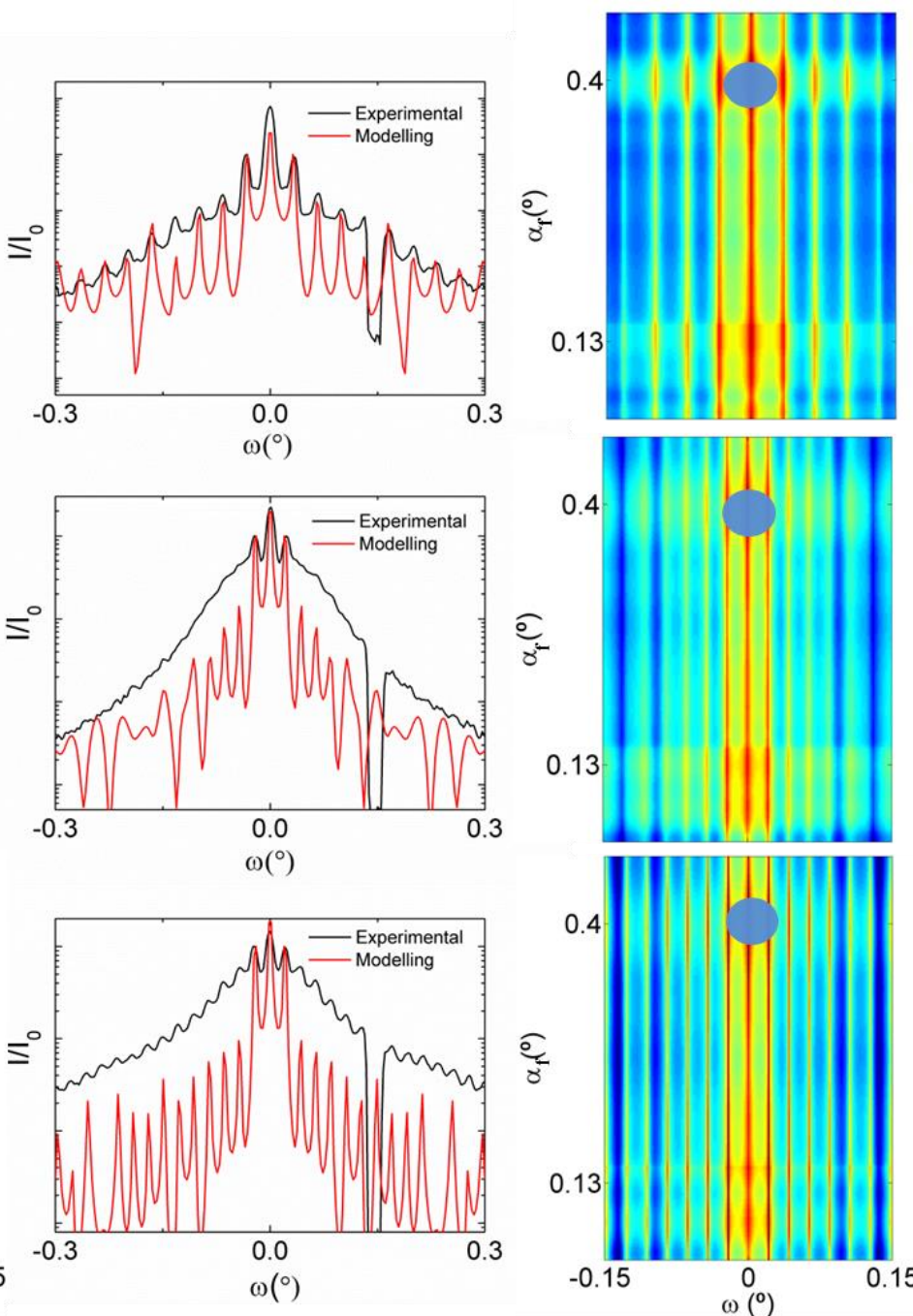

(b)

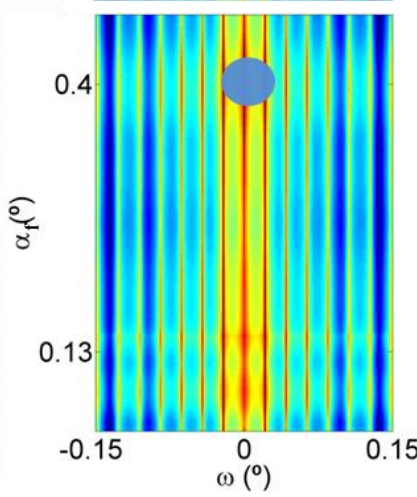

(c)

Figure 7. (a) Experimental GISAXS patterns of PBAC films irradiated at $266 \mathrm{~nm}$ with 1200 pulses at different regions of the laser irradiated spot, as labeled according to Fig. 2a. (b) Experimental (black lines) and simulated (red lines) GISAXS intensity profiles taken at a fixed exit angle $\alpha_{f}=0.13^{\circ}$. (c) Corresponding simulated GISAXS patterns.

\section{CONCLUSIONS}

An accurate mapping of the structural order of LIPSS in polymer thin films has been carried out by accomplishing a microfocus beam GISAXS scan, Atomic Force Microscopy imaging and GISAXS modelling across the diameter of the laser beam perpendicular to the laser 
polarization vector. This combined study has allowed evaluating the effects on structure formation of the non-homogeneous local laser pulse energy, mapping with micrometric resolution the evolution of the period and degree of structural order of LIPSS. Regions of LIPSS with different period and degree of structural order have been identified and associated with their respective local laser energies. The experiments presented here go one step further towards controlling LIPSS formation through a deep understanding of the parameters that influence the nanostructure formation process.

\section{AUTHOR INFORMATION}

\section{Corresponding Author}

*E-mail: i.martin-fabiani@surrey.ac.uk

\section{ACKNOWLEDGMENT}

The authors acknowledge financial support from MINECO (grants MAT2009-07789, CTQ2010-15680, CTQ2013-43086, MAT2011-23455 and MAT2012-33517, FPI BES-2010030074 and Ramón y Cajal contract RYC-2011-08069). The assistance of S. Roth at PO3 (PETRA III) is greatly acknowledged.

\section{REFERENCES}

(1) Chen, D.; Zhao, W.; Russell, T. P. P3ht Nanopillars for Organic Photovoltaic Devices Nanoimprinted by Aao Templates. ACS Nano 2012, 6, 1479-1485.

(2) Xia, L.; Wei, Z. X.; Wan, M. X. Conducting Polymer Nanostructures and Their Application in Biosensors. J. Colloid Interface Sci. 2010, 341, 1-11.

(3) Leiprecht, P.; Kuehler, P.; Longo, M.; Leiderer, P.; Afonso, C. N.; Siegel, J. Exploiting Optical near Fields for Phase Change Memories. Appl. Phys. Lett. 2011, 98, 013103.1-3. 
(4) P. Müller-Buschbaum; E. Bauer; S. Pfister; S. V. Roth; M. Burghammer; C. Riekel; C. David; U. Thiele. Creation of Multi-Scale Stripe-Like Patterns in Thin Polymer Blend Films. Europhys. Lett. 2006, 73, 35.

(5) Müller-Buschbaum, P.; Bauer, E.; Maurer, E.; Schlögl, K.; Roth, S. V.; Gehrke, R. Route to Create Large-Area Ordered Polymeric Nanochannel Arrays. Appl. Phys. Lett. 2006, 88, 083114.1-3.

(6) Zhang, P.; Huang, H.-y.; Chen, Y.; Yu, S.; Krywka, C.; Vayalil, S.; Roth, S.; He, T.-b. Preparation of Long-Range Ordered Nanostructures in Semicrystalline Diblock Copolymer Thin Films Using Micromolding. Chin. J. Polym. Sci. 2014, 32, 1188-1198.

(7) Rueda, D. R.; Martin-Fabiani, I.; Soccio, M.; Alayo, N.; Perez-Murano, F.; Rebollar, E.; Garcia-Gutierrez, M. C.; Castillejo, M.; Ezquerra, T. A. Grazing-Incidence Small-Angle XRay Scattering of Soft and Hard Nanofabricated Gratings. J. Appl. Crystallogr. 2012, 45, 1038-1045.

(8) Brown, M.; Arnold, C., Fundamentals of Laser-Material Interaction and Application to Multiscale Surface Modification. In Laser Precision Microfabrication, Sugioka, K.; Meunier, M.; Piqué, A., Eds.; Springer Berlin Heidelberg: 2010, Vol. 135, pp 91-120.

(9) Muller-Buschbaum, P. Grazing Incidence Small-Angle X-Ray Scattering: An Advanced Scattering Technique for the Investigation of Nanostructured Polymer Films. Anal. Bioanal. Chem. 2003, 376, 3-10.

(10) Renaud, G.; Lazzari, R.; Leroy, F. Probing Surface and Interface Morphology with Grazing Incidence Small Angle X-Ray Scattering. Surf. Sci. Rep. 2009, 64, 255-380.

(11) Schwartzkopf, M.; Buffet, A.; Korstgens, V.; Metwalli, E.; Schlage, K.; Benecke, G.; Perlich, J.; Rawolle, M.; Rothkirch, A.; Heidmann, B.; Herzog, G.; Muller-Buschbaum, P.; Rohlsberger, R.; Gehrke, R.; Stribeck, N.; Roth, S. V. From Atoms to Layers: In Situ Gold Cluster Growth Kinetics During Sputter Deposition. Nanoscale 2013, 5, 5053-5062. 
(12) Yu, S.; Santoro, G.; Sarkar, K.; Dicke, B.; Wessels, P.; Bommel, S.; Döhrmann, R.; Perlich, J.; Kuhlmann, M.; Metwalli, E.; Risch, J. F. H.; Schwartzkopf, M.; Drescher, M.; Müller-Buschbaum, P.; Roth, S. V. Formation of Al Nanostructures on Alq3: An in Situ Grazing Incidence Small Angle X-Ray Scattering Study During Radio Frequency Sputter Deposition. J. Phys. Chem. Lett. 2013, 4, 3170-3175.

(13) Roth, S. V.; Burghammer, M.; Riekel, C.; Müller-Buschbaum, P.; Diethert, A.; Panagiotou, P.; Walter, H. Self-Assembled Gradient Nanoparticle-Polymer Multilayers Investigated by an Advanced Characterization Method: Microbeam Grazing Incidence X-Ray Scattering. Appl. Phys. Lett. 2003, 82, 1935-1937.

(14) Lazzari, R. Isgisaxs: A Program for Grazing-Incidence Small-Angle X-Ray Scattering Analysis of Supported Islands. J. Appl. Crystallogr. 2002, 35, 406-421.

(15) Babonneau, D. Fitgisaxs: Software Package for Modelling and Analysis of Gisaxs Data Using Igor Pro. J. Appl. Crystallogr. 2010, 43, 929-936.

(16) Meier, R.; Chiang, H.-Y.; Ruderer, M. A.; Guo, S.; Koerstgens, V.; Perlich, J.; MuellerBuschbaum, P. In Situ Film Characterization of Thermally Treated Microstructured Conducting Polymer Films. J. Polym. Sci., Part B: Polym. Phys. 2012, 50, 631-641.

(17) Maret, M.; Liscio, F.; Makarov, D.; Doisneau-Cottignies, B.; Ganss, F.; Missiaen, J. M.; Albrecht, M. Growth Temperature Effect on the Structure of Copt Islands on Nacl(001) Studied by Grazing-Incidence Small-Angle X-Ray Scattering. J. Appl. Crystallogr. 2014, 47, 102-109.

(18) Guosheng, Z.; Fauchet, P. M.; Siegman, A. E. Growth of Spontaneous Periodic SurfaceStructures on Solids During Laser Illumination. Phys. Rev. B 1982, 26, 5366-5381.

(19) Bäuerle, D. In Laser Processing and Chemistry, ed.; Springer-Verlag: Berlin, 2000.

(20) Csete, M.; Bor, Z. Laser-Induced Periodic Surface Structure Formation on PolyethyleneTerephthalate. Appl. Surf. Sci. 1998, 133, 5-16. 
(21) Bolle, M.; Lazare, S. Large-Scale Excimer-Laser Production of Submicron Periodic Structures on Polymer Surfaces. Appl. Surf. Sci. 1993, 69, 31-37.

(22) Martin-Fabiani, I.; Rebollar, E.; Perez, S.; Rueda, D. R.; Garcia-Gutierrez, M. C.; Szymczyk, A.; Roslaniec, Z.; Castillejo, M.; Ezquerra, T. A. Laser-Induced Periodic Surface Structures Nanofabricated on Poly(Trimethylene Terephthalate) Spin-Coated Films. Langmuir 2012, 28, 7938-7945.

(23) Rebollar, E.; Vazquez de Aldana, J. R.; Martin-Fabiani, I.; Hernandez, M.; Rueda, D. R.; Ezquerra, T. A.; Domingo, C.; Moreno, P.; Castillejo, M. Assessment of Femtosecond Laser Induced Periodic Surface Structures on Polymer Films. Phys. Chem. Chem. Phys. 2013, 15, 11287-11298.

(24) Rebollar, E.; Sanz, M.; Perez, S.; Hernandez, M.; Martin-Fabiani, I.; Rueda, D. R.; Ezquerra, T. A.; Domingo, C.; Castillejo, M. Gold Coatings on Polymer Laser Induced Periodic Surface Structures: Assessment as Substrates for Surface-Enhanced Raman Scattering. Phys. Chem. Chem. Phys. 2012, 14, 15699-15705.

(25) Rebollar, E.; Perez, S.; Hernandez, J. J.; Martin-Fabiani, I.; Rueda, D. R.; Ezquerra, T. A.; Castillejo, M. Assessment and Formation Mechanism of Laser-Induced Periodic Surface Structures on Polymer Spin-Coated Films in Real and Reciprocal Space. Langmuir 2011, 27, $5596-5606$.

(26) Rebollar, E.; Perez, S.; Hernandez, M.; Domingo, C.; Martin, M.; Ezquerra, T. A.; Garcia-Ruiz, J. P.; Castillejo, M. Physicochemical Modifications Accompanying Uv Laser Induced Surface Structures on Poly(Ethylene Terephthalate) and Their Effect on Adhesion of Mesenchymal Cells. Phys. Chem. Chem. Phys. 2014, 16, 17551-17559.

(27) Buffet, A.; Rothkirch, A.; Dohrmann, R.; Korstgens, V.; Kashem, M. M. A.; Perlich, J.; Herzog, G.; Schwartzkopf, M.; Gehrke, R.; Muller-Buschbaum, P.; Roth, S. V. P03, the 
Microfocus and Nanofocus X-Ray Scattering (Minaxs) Beamline of the Petra Iii Storage Ring: The Microfocus Endstation. J. Synchrotron Radiat. 2012, 19, 647-653.

(28) Hammersley, A. Fit2d: An Introduction and Overview; 1997.

(29) Hindeleh, A. M.; Hosemann, R. Microparacrystals - the Intermediate Stage between Crystalline and Amorphous. J. Mater. Sci. 1991, 26, 5127-5133. 


\title{
Mapping the Structural Order of Laser Induced
}

\author{
Periodic Surface Structures in Thin Polymer Films
}

\section{by Microfocus Beam Grazing Incidence Small}

\section{Angle X-Ray Scattering}

Ignacio Martín-Fabiani, *,1 Esther Rebollar, ${ }^{2}$ Mari Cruz García-Gutiérrez, ${ }^{3}$ Daniel R. Rueda, ${ }^{3}$ Marta Castillejo, ${ }^{2}$ and Tiberio A. Ezquerra ${ }^{3}$

${ }^{1}$ Department of Physics, University of Surrey, Guildford GU2 7XH, United Kingdom

${ }^{2}$ Instituto de Química Física Rocasolano (IQFR-CSIC), Serrano 119, 28006 Madrid, Spain

${ }^{3}$ Instituto de Estructura de la Materia (IEM-CSIC), Serrano 121, 28006 Madrid, Spain

TABLE OF CONTENTS

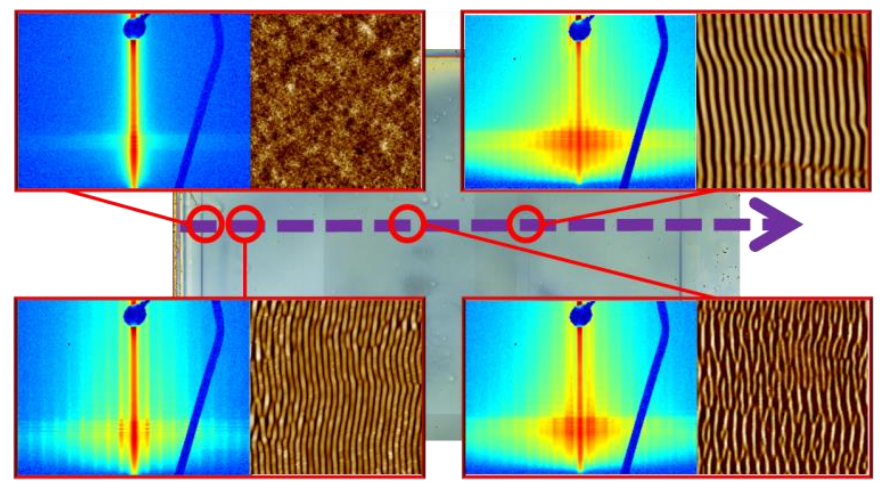

